\title{
Role of sawtooth in avoiding impurity accumulation and maintaining good confinement in JET radiative mantle discharges
}

\author{
M.F.F. Nave ${ }^{1}$, J. Rapp ${ }^{2}$, T. Bolzonella ${ }^{3}$, R. Dux ${ }^{4}$, M.J. Mantsinen ${ }^{5}$, R. Budny ${ }^{6}$, \\ P. Dumortier ${ }^{7}$, M. von Hellermann ${ }^{8}$, S. Jachmich ${ }^{2}$, H.R. Koslowski ${ }^{2}$, \\ G. Maddison ${ }^{9}$, A. Messiaen ${ }^{7}$, P. Monier-Garbet ${ }^{10}$, J. Ongena ${ }^{7}$, M.E. Puiatti ${ }^{3}$, \\ J. Strachan ${ }^{6}$, G. Telesca ${ }^{7}$, B. Unterberg ${ }^{2}$, M. Valisa ${ }^{3}$, P. de Vries ${ }^{8,9}$ and \\ contributors to the JET-EFDA Workprogramme ${ }^{a}$ \\ ${ }^{1}$ Associação EURATOM/IST, Centro de Fusão Nuclear, 1049-001 Lisbon, Portugal \\ ${ }^{2}$ Association EURATOM-FZ-Juelich, Institut fuer Plasmaphysik, TEC, 52425 Juelich, Germany \\ ${ }^{3}$ Consorzio RFX-Associazione Euratom-Enea sulla Fusione, Corso Stati Uniti 4, I-35127 Padova, Italy \\ ${ }^{4}$ Max-Planck-Institut für Plasmaphysik, IPP-EURATOM Assoziation, Boltzmann-Str. 2, D-85748 Garching, \\ Germany \\ ${ }^{5}$ Helsinki University of Technology, Association Euratom-Tekes, PO Box 2200, FIN-02015 HUT, Finland \\ ${ }^{6}$ PPPL, Princeton University, NJ, USA \\ ${ }^{7}$ LPP-ERM/KMS, Association Euratom-Belgian State, Brussels, Belgium \\ ${ }^{8}$ Associatie EURATOM-FOM, Rijnhuizen, PO Box 1207, 3430 BE, Netherlands \\ ${ }^{9}$ Euratom/UKAEA Fusion Association, Culham Science Centre, Abingdon, OX14 3DB, UK \\ ${ }^{10}$ Association EURATOM/CEA CEA CADARACHE, DRFC, Bâtiment 513, 13108 Saint-Paul-Lez-Durance, \\ France
}

Received 13 May 2002, accepted for publication 2 September 2003

Published 26 September 2003

Online at stacks.iop.org/NF/43/1204

\begin{abstract}
Impurity injection in the JET ELMy H-mode regime has produced high-confinement, quasi-steady-state plasmas with densities close to the Greenwald density. However, at large Ar densities, a sudden loss of confinement is observed. A possible correlation between loss of confinement and the observed MHD phenomena, both in the core and in the edge of the plasma, was considered. The degradation in confinement coincided with impurity profile peaking following the disappearance of sawtooth activity. In addition, impurity density profile analysis confirmed that central MHD modes prevented impurity peaking. Experiments were designed to understand the role of sawtooth crashes in re-distributing impurities. Ion-cyclotron radio frequency heating was used to control the central $q$-profile and maintain sawtooth activity. This resulted in quasi-steady-state, high-performance plasmas with high Ar densities. At $H_{98 y} * f_{\mathrm{GWD}} \sim 0.8$ and high Ar injection rates, quasi-steady-states, which previously only lasted $<1 \tau_{E}$, were now maintained for the duration of the heating $\left(\Delta t \sim 9 \tau_{E}\right)$. The increased central heating may have an additional beneficial effect in opposing impurity accumulation by changing the core power balance and modifying the impurity transport as predicted by neo-classical theory.
\end{abstract}

PACS numbers: 52.55.Fa, 52.55.Tn, 52.25.Vy

\section{Introduction}

Injection of high- $Z$ impurities to create a cool radiative mantle around the plasma has been a successful technique

a See appendix of the paper by Pamela J. 2000 Overview of recent JET results Proc. IAEA Conf. on Fusion Energy (Sorrento, 2000). used in the TEXTOR [1-3] and DIII-D [4-7] tokamaks to improve the level of confinement in high-density regimes. Experiments performed at JET [8-10] and JT60-U [11,12] in recent years have tested this technique in larger machines. JET radiative mantle experiments in ELMy H-mode regimes produced high-confinement, quasi-steady-state plasmas with 
high-confinement $\left(H_{98 y} \sim 1\right)$ and densities close to the Greenwald density $\left(n / n_{\mathrm{GWD}} \geqslant 0.85\right)[9]$ as required for the future operation of ITER [13].

JET radiative mantle experiments have been mainly performed in divertor plasmas, following two main lines of thought: (a) L-mode plasmas, heated by neutral beam injection (NBI) before the plasma current is fully penetrated [14], as in DIII-D experiments [4-6]; and (b) ELMy H-mode plasmas, heated by NBI during the plasma current flat top [9]. This paper describes the work done to understand the loss of confinement and the measures taken to improve stationarity of ELMy $\mathrm{H}$-mode discharges with high radiation fractions $\left(f_{\text {rad }}=P_{\text {rad }} / P_{\text {tot }}=0.5-0.65\right.$, increasing to $f_{\text {rad }}=1$ during ELM-free periods) using argon (Ar) injection. We consider low-triangularity $(\delta \sim 0.2)$ discharges obtained in the septum configuration [15], i.e. discharges with the X-point embedded in the dome of the JET MKII-GB divertor [16].

The septum radiative mantle experiments of JET share some characteristics with the limiter radiative improved (RI) modes of TEXTOR [1]. In JET, the dome of the divertor played the role of a limiter. As in TEXTOR, JET experiments show that higher densities can be achieved in discharges with an increased radiation level, when compared with discharges without impurity injection [9]. However, unlike TEXTOR, where impurity seeding leads to density and confinement increases (up to $30 \%$ confinement improvements were observed), at JET $H_{98 y} \sim 1$ is maintained, but at higher densities. With larger impurity injection rates $\left(\phi_{\mathrm{Ar}} \gtrsim\right.$ $\left.4 \times 10^{21} \mathrm{el} \mathrm{s}^{-1}\right)$, confinement is reduced. The aim of the paper is to investigate the cause of confinement degradation and to propose a solution.

One TEXTOR RI-mode characteristic was the peaked plasma current distribution compared with the unseeded case (central $q$-factor $(q(0))$ could be as small as 0.6) [17]. When impurity puffing ended, $q(0)$ increased, and after a transient improvement, confinement decreased. Both JET and TEXTOR experiments indicated that control of the central current density and core MHD stability may maintain the good confinement phase. Central MHD activities in TEXTOR RImodes [17] and JET radiative mantle ELMy H-modes [18] are similar. This paper shows that by controlling $q(0)$ and core MHD activity, the good confinement phase of JET radiative mantle discharges was prolonged. One difference between the TEXTOR and JET experiments was the edge conditions. TEXTOR confinement improvement arises from the plasma core, while JET radiative mantle experiments combine good core confinement with edge $\mathrm{H}$-mode confinement. Therefore, edge stability and ELM behaviour were important in JET plasmas and contribute to maintaining good confinement.

Section 2, below describes observations in septum discharges with high impurity densities. The correlation between confinement loss and MHD phenomena are described for the core in section 3 and the edge of the plasma in section 4 . The degradation correlated with two main phenomena: (a) $\mathrm{Ar}$ accumulation in the plasma core and (b) disappearance of sawtooth activity. Experiments designed to understand the role of sawtooth crashes in re-distributing impurities are reported in section 5. Control of sawteeth activity with ion-cyclotron radio frequency heating (ICRH) resulted in a quasi-steadystate while maintaining high-performance. Further benefits of increasing heating in the plasma core are discussed in section 6. Power balance results for discharges with and without ICRH are compared with predictions from the neo-classical impurity accumulation model of [19]. Conclusions are presented in section 7 .

\section{Confinement loss at high impurity injection}

In the septum configuration, the highest-performance plasmas $\left(H_{98 y} * f_{\mathrm{GWD}} \geqslant 0.8\right)$ were obtained with $12 \mathrm{MW}$ NBI heating, with two gas injection phases: an initial phase of continuous $\mathrm{D}_{2}$ and Ar fuelling, followed by the 'after-puff' phase when both gases' injection rates were reduced. The high-confinement was obtained in the after-puff phase. At the start of the after-puff phase, confinement improved for 1-2 s, then either saturated or declined (e.g. at $1.2 \mathrm{~s}$ in figure $1(\mathrm{~g})$ ). In discharges with low or moderate Ar seeding, a quasi-steady-state regime remained through to the end of the applied heating [9]. However, at higher $\mathrm{Ar}$ injection rates $\left(\phi_{\mathrm{Ar}} \geqslant 4 \times 10^{21} \mathrm{el} \mathrm{s}^{-1}\right)$, the high-confinement phase was transient (figure $1(g)$ ).

In the puff phase (figure 1), high deuterium fuelling was used to increase the density, while the radiation level was also increased by Ar injection. At this high level of fuelling, the energy confinement time decreased. In the after-puff phase, the $\mathrm{D}_{2}$ puff was reduced or stopped, while Ar injection continued at a lower level. The energy content (figure $1(f)$ ) was higher than in its previous H-mode level. Several MHD changes occurred in the after-puff phase. In the core, the sawtooth

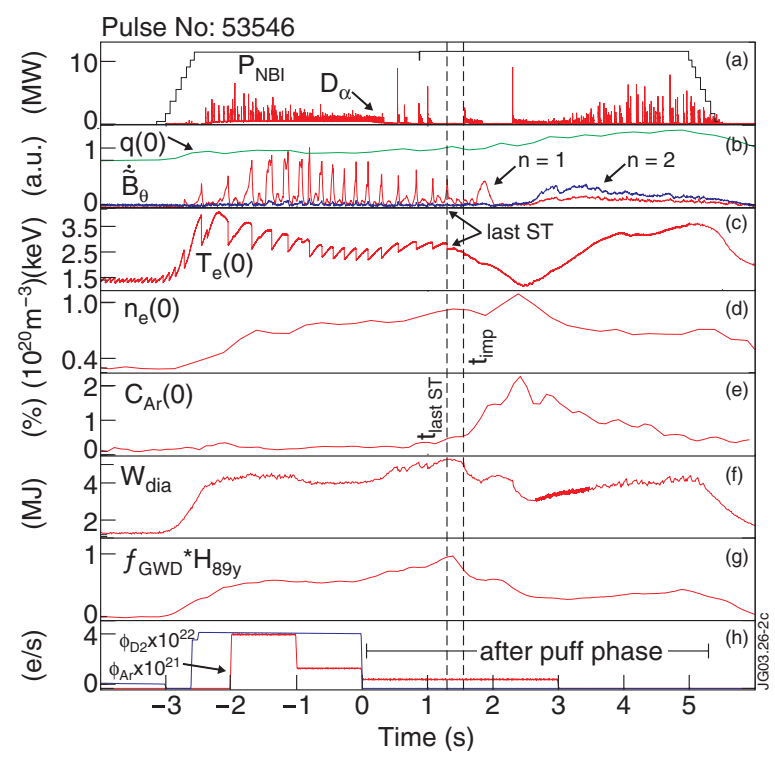

Figure 1. Overview of a discharge with high argon injection rate $\left(P_{\mathrm{NBI}}=12 \mathrm{MW}, I_{\mathrm{p}}=2.5 \mathrm{MA}, B_{\mathrm{T}}=2.5 \mathrm{~T}\right)$. The boxes contain the following parameters plotted versus time, where $t=0 \mathrm{~s}$ is the start of the after-puff phase: $(a)$ heating power and $\mathrm{D}_{\alpha}$ emission showing the ELM behaviour. (b) $q$ on-axis from EFIT, $n=1$ and 2 magnetic perturbations. The spikes in the $n=1$ signal are sawtooth MHD precursors. (c) Central electron temperature. (d) Central electron density. $(e)$ Central argon concentration from bolometry analysis. $(f)$ Plasma stored energy. $(g)$ The product $f_{\mathrm{GWD}} * H_{98 y}$.

(h) Deuterium and argon injection rates. In this discharge, a low $\mathrm{Ar}$ rate is maintained in the after-puff phase. The first dashed line indicates the time of the last sawtooth, the second dashed line indicates $t_{\text {imp }}$, the time of sudden impurity inflow. 
amplitude decreased, as normally observed at a high core density. The sawtooth crashes stop (1.3 s in figure 1$)$. The central temperature signal and the magnetic pick-up coil signal, where the spikes corresponding to sawtooth precursors are observed, were indicators of sawtooth MHD activity. Edge stability also changes. The type III ELM regime observed during the puff phase changes to type I ELMs. (The ELM types in this paper are the same as defined in [20].) In the afterpuff phase, energy confinement degradation coincided with $\mathrm{Ar}$ accumulation in the plasma core. In figure 1 the central Ar concentration, $C_{\mathrm{Ar}}(0)$, starts to rise slowly at $1.2 \mathrm{~s}$, with a sudden larger increase, $\Delta C_{\mathrm{Ar}}(0) / \Delta t=2 \% \mathrm{~s}^{-1}$, observed at $1.5 \mathrm{~s}$ (indicated in figure 1 as $t_{\mathrm{imp}}$ ). Further energy confinement loss occurred due to core radiative collapse ( $2.3 \mathrm{~s}$ in figure $1(f)$, discussed later in section 6).

The phenomenology was similar at lower impurity injection rates. Impurity density peaking and sawtooth suppression were observed (see section 3 ). With lower central impurity concentrations [9], the confinement degradation was more steady at a value about $10 \%$ below the no-Ar values [9].

The impurity accumulation correlated in time with both the sawtooth suppression and the ELM changes (figure 1). This suggested that the central impurity peaking could be correlated with either or both of the changes in central and edge MHD activity. Analysis of impurity densities from many discharges indicated that impurity peaking occurred independent of ELM repetition frequency (see section 4 ), while a correlation was found between impurity peaking and sawtooth suppression (section 3).

The temporal evolution of the central Ar density was derived from bolometry measurements for 50 discharges. The bolometer signals in the upper half of the poloidal cross section were Abel inverted with the assumption of a poloidally symmetric radiation distribution. (The Ar was injected from the top of the machine.) The core Ar was assumed to be in coronal equilibrium. Thus the $\mathrm{Ar}$ concentration on the axis, $C_{\mathrm{Ar}}(0)$, was $C_{\mathrm{Ar}}(0)=P_{\mathrm{rad}}(0) /\left(n_{\mathrm{e}}(0)^{2} L_{\mathrm{u}}\left(T_{\mathrm{e}}(0)\right)\right)$ using the cooling rates, $L_{\mathrm{u}}$, from Post [21], where $n_{\mathrm{e}}$ is the electron density, $T_{\mathrm{e}}$ is the electron temperature and $P_{\mathrm{rad}}$ is the radiated power density.

For a smaller number of discharges, with low to moderate Ar levels, impurity density profiles were obtained from analysis of soft $\mathrm{x}$-ray (SXR) emission profiles, as explained in the next section.

\section{Impurity peaking and sawtooth observations}

The sawtooth period and amplitude observed in the radiative mantle discharges followed the empirical relations obtained for non-seeded JET H-modes with NBI heating, described in [22]. The scaling of sawtooth properties with plasma parameters has been obtained at JET by a regression analysis from over 300 discharges, indicating that the sawtooth period is proportional to: $T_{\mathrm{e}}^{1.7} n_{\mathrm{e}}^{0.23}$, while the amplitude was proportional to: $n_{\mathrm{e}}^{-0.53} q_{95}^{-0.98} p_{\mathrm{p}}^{0.23} \delta^{0.23}(\kappa-1)^{-1.8}$ (where $p_{\mathrm{p}}$ is the pressure profile peaking factor, $n_{\mathrm{e}}$ and $T_{\mathrm{e}}$ are the central electron density and temperature, $\delta$ is the average triangularity and $\kappa$ is the elongation) [22]. The reduction in sawtooth amplitude at a higher density also occurred in the absence of $\operatorname{Ar}[22]$.

Slow impurity accumulation in the plasma core was observed when sawtooth crashes had a reduced amplitude. In discharges with a high Ar input, as in figure 1, a sudden increase in central impurity concentration, $\Delta C_{\mathrm{Ar}}(0) / \Delta t \geqslant 1 \% \mathrm{~s}^{-1}$, followed the disappearance of sawtooth activity. The time of sudden impurity accumulation $\left(t_{\text {imp }}\right.$ in figure 1$)$ equalled the time of the last sawtooth crash within $\pm 0.3 \mathrm{~s}$ with a range of $5 \mathrm{~s}$ (figure 2). In most cases, this increase in $C_{\mathrm{Ar}}(0)$ was observed in the after-puff phase; however, in a few cases, both sawtooth suppression and impurity accumulation were observed in the puffing phase.

Central radiation increase and impurity accumulation were not seen in discharges where central MHD modes, sawteeth and/or continuous ( $m=1, n=1)$ modes were observed up to the end of the after-puff phase. With $P_{\mathrm{NBI}}=$ $12 \mathrm{MW}$, central $n=1$ modes remaining to the end of the heating phase were seen in unseeded discharges and occasionally in seeded discharges with a low Ar injection rate. Two discharges with low Ar injection rate $\left(\phi_{\mathrm{Ar}} \sim\right.$ $\left.1 \times 10^{21} \mathrm{el} \mathrm{s}^{-1}\right)$ that showed different sawtooth behaviour are compared in figure 3 . With the sawteeth activity maintained (figure 3, top), no central radiation peaking is observed. In figure 3 (bottom), sawteeth are suppressed at $t \sim 2 \mathrm{~s}$ whilst a continuous central $(m=1, n=1)$ lasts until $t \sim 3.5 \mathrm{~s}$. The central radiation started to increase at the disappearance of the sawtooth. The radiation profile became more peaked once the central $n=1$ mode disappeared. (Although, $P_{\text {rad }}$ became peaked, central impurity accumulation was not observed since in this case the Ar content is very low.)

The observation that sawtooth-free periods coincide with core impurity accumulation was similar to observations from ASDEX [23], ASDEX-Upgrade [24, 25] and TEXTOR [26]. In ASDEX-Upgrade the absence of sawtooth and fishbone relaxations correlated with an increase in central SXR emission and indicated an impurity density increase. Those experiments suggested that sawteeth and other central MHD modes

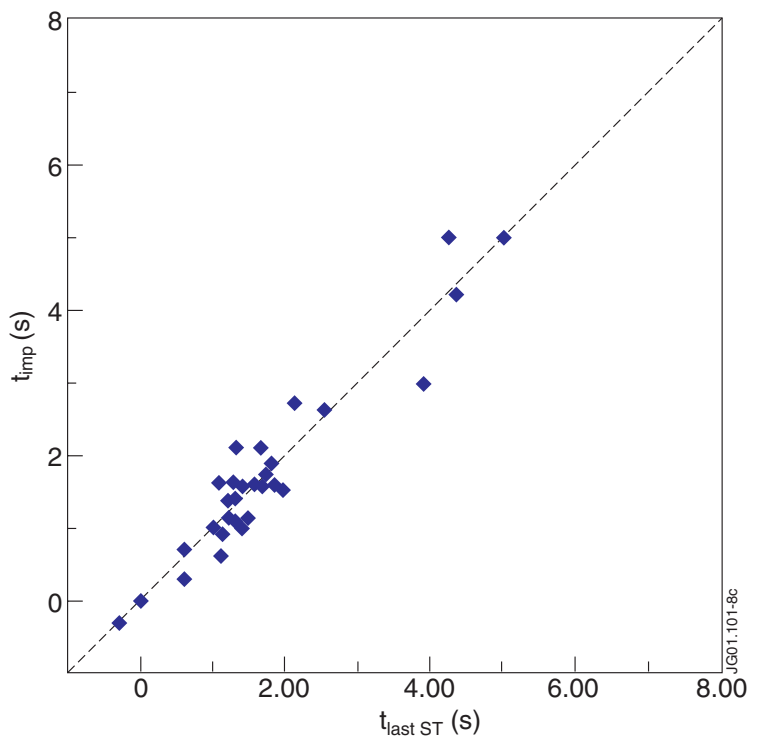

Figure 2. Time of sudden increase in central impurity concentration versus time the last sawtooth crash is observed (see $t_{\text {imp }}$ and $t_{\text {last ST }}$, indicated by dashed lines in figure 1). In this figure, only the cases where $C_{\mathrm{Ar}}(0)$ increases at a rate $\geqslant 1.0 \% \mathrm{~s}^{-1}$ were considered. For $t \geqslant 5 \mathrm{~s}$, sawteeth were maintained throughout the heating (up to $8 \mathrm{~s}$ ) and no impurity peaking was observed. 


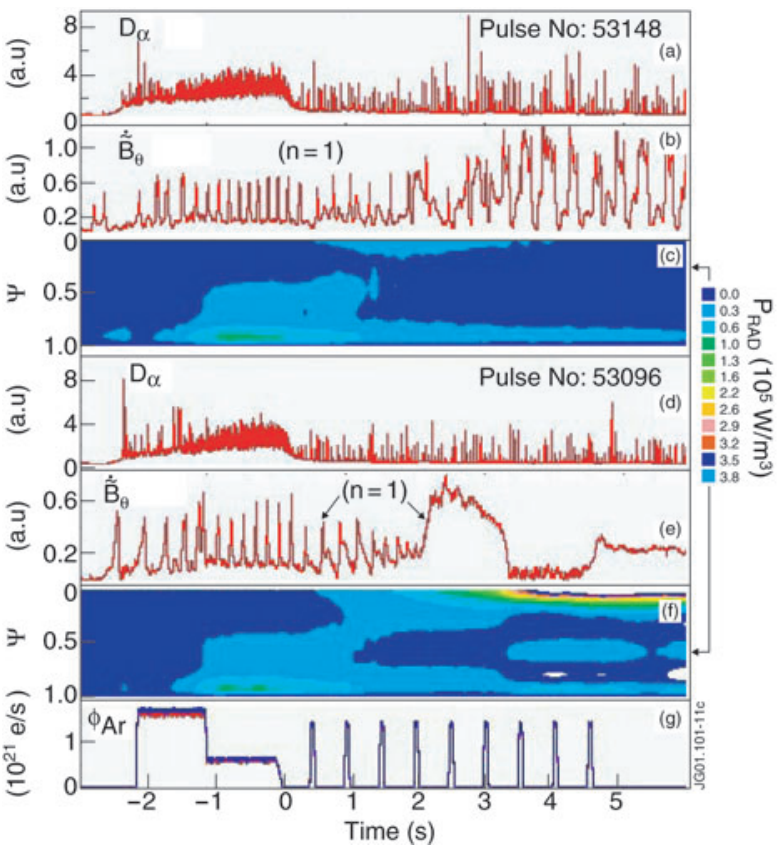

Figure 3. Comparison of discharges with low Ar injection rates showing different sawtooth observations in the after-puff phase. The first three boxes $(a)-(c)$ show: the temporal evolution of $\mathrm{D}_{\alpha}$ emission, magnetic pick-up coil signal, contour plot of the radiation power density (Abel inverted bolometer profiles, $\Psi$ is the square root of the normalized poloidal flux function) for discharge 53148, whilst the next three boxes $(d)-(f)$ show the same quantities for discharge 53096. Both discharges have the same Ar rate $(g)$. Time $=0 \mathrm{~s}$ is the start of the after-puff phase. In discharge 53148 with sawtooth crashes until the end of the heating phase, radiation peaking is not observed $(c)$. In discharge 53096 with sawtooth suppression at $t=2 \mathrm{~s}$, radiation peaking is observed. The peak value $\left(P_{\mathrm{RAD}}=4.8 \times 10^{6} \mathrm{~W} \mathrm{~m}^{-3}\right)$ is outside the plotted scale $(f)$.

contributed to impurity profile control. SXR analysis of JET radiative mantle discharges, reported here, lead to similar conclusions.

Typically, in the puff phase, the central $q$-value is below unity, $q(0) \sim 0.9$ (EFIT code equilibrium reconstruction [27]). Once the gas rate was decreased, $q(0)$ increased and sawtooth suppression occurred when $q(0)$ rose above unity (figure 1$)$. The $q(0)$ rose due to an increase in edge pedestal temperature in the after-puff phase. The resistivity at the plasma edge decreased and the plasma current was re-distributed, leading to an increased edge plasma current density and consequently to a reduced central plasma current density. In contrast, the current density profile became very peaked during the TEXTOR RI-mode high-confinement phase [17], due to the radiating belt with an L-mode edge. However, also at TEXTOR when the impurity seeding was stopped, $q(0)$ was in some cases observed to rise above unity [17].

In JET the magnetic equilibrium reconstruction as well as MHD mode analysis [18] indicated that the central $q$-profile was nearly flat and close to unity near the time of sawtooth suppression. After sawtooth suppression, equilibrium reconstruction with polarimetric measurements [28] indicated reversed shear $q$-profiles (figure 4). The evolution for $q(0)$, after it rises above unity, not observed in non-seeded discharges, was plausibly a result of impurity accumulation. When the central $q$-profile was flat and close
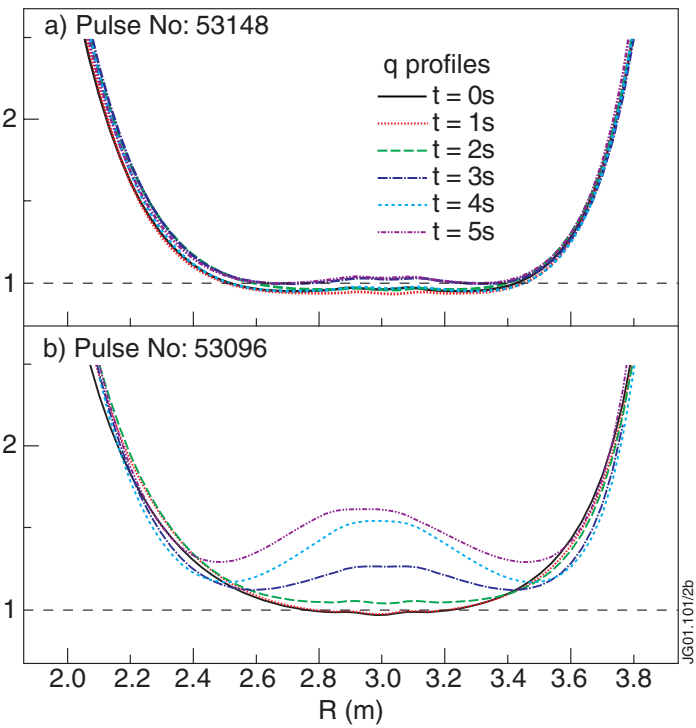

Figure 4. Magnetic equilibrium reconstruction including polarimetric measurements for the two discharges shown in figure 3 . In the discharge with sawteeth present throughout the heating phase, the central $q$ is nearly flat with $q(0) \sim 1$. In the discharge with sawtooth suppression, reversed shear $q$-profiles are obtained after the sawtooth crashes stop (as with observations in TEXTOR [17]).

to unity, the ( $m=1, n=1)$ precursor mode associated with sawteeth was a continuous mode (figure 3, top, $t>2 \mathrm{~s}$ ). The core MHD modes observed are similar to those reported from TEXTOR [17] when $q(0)$ was close to unity. Reversed shear, associated with impurity accumulation, was also seen in TEXTOR $[17,26]$ in accumulation studies of high- $Z$ impurities in radiation-cooled discharges. In JET, if the central $q$ is non-monotonic and above unity, $(m=4, n=3)$ and ( $m=3, n=2)$ modes may become unstable. The $(m=3$, $n=2)$ modes limited core density [18].

On-axis ICRH (figures 5 and 6) altered the $q(0)$ evolution so that the sawtooth instability was maintained throughout the heating phase in discharges with moderate to high impurity injection (section 5). The increased sawtooth period due to ICRH fast-particle effects allowed us to study the effect of sawtooth crashes on the evolution of the Ar profiles, obtained from SXR analysis, in a discharge where the sawtooth repetition frequency was quite low. The sawtooth crash re-distributed impurities (figure 5). In the fuelling phase $(-1$ to $0 \mathrm{~s}$ in figure 5), the central impurity density increased at each sawtooth crash. During the early phase, the Ar impurity profiles were hollow. A similar phenomenon was observed during laser ablation experiments in JET L-mode and H-mode ELM-free plasmas, where SXR analysis indicated that sawtooth crashes facilitated impurity penetration into the plasma core $[29,30]$. Later, sawtooth crashes correlated with flat profiles inside $\Psi=0.5$ and reduced peaking comparing the centre with $\Psi=0.8(t=1-3 \mathrm{~s}$ in figure 5). Impurity flattening after a sawtooth crash has been observed in several machines and with a large variety of impurities [31-35]. It was seen previously at JET in laser ablation experiments in ohmically heated plasmas where the confinement time of the impurity ions was several times longer than the sawtooth period [36]. Continuous core MHD modes (seen in figure 6 , in between sawtooth crashes) also correlated with reduced core impurity 


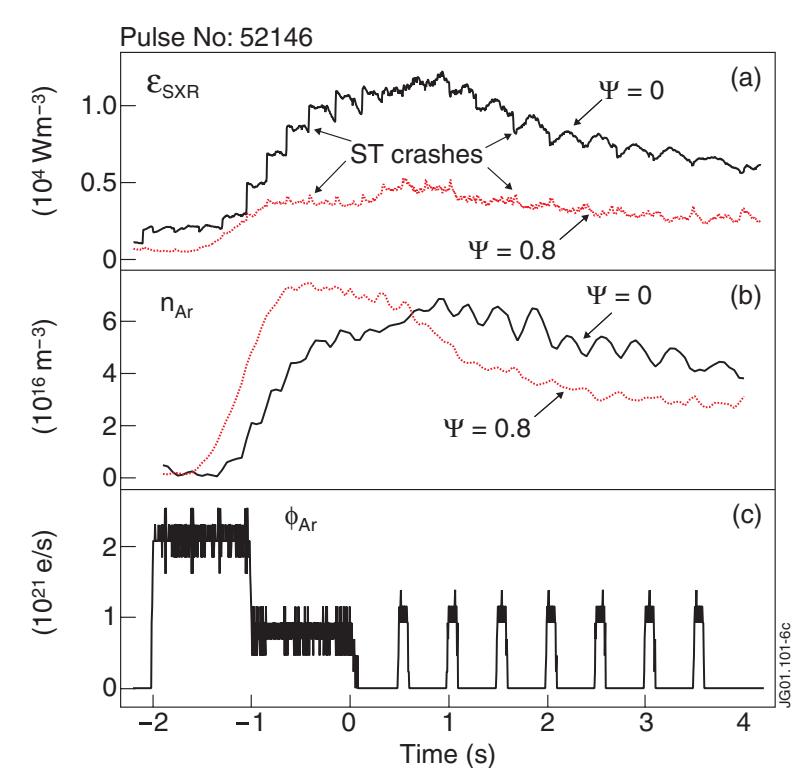

Figure 5. (a) Central and edge SXR emissivity and (b) corresponding impurity density deduced from SXR data plotted versus time, for a discharge with moderate argon injection rate $(c)$. In this discharge sawteeth were maintained by adding $3 \mathrm{MW}$ of ICRF heating to $12 \mathrm{MW}$ NBI heating. In the puff phase the central SXR emissivity showed inverted sawteeth, indicating an increase in the impurity level. In the after-puff phase, sawtooth crashes flattened the impurity density profile.

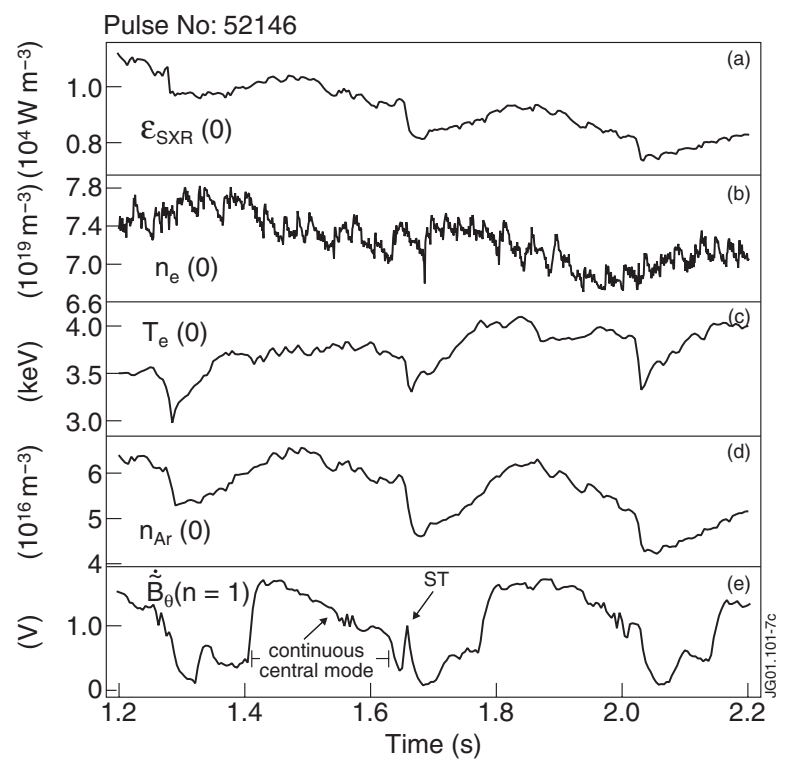

Figure 6. Same discharge as in figure 5. Central values of SXR emissivity $(a)$, electron density $(b)$ and electron temperature $(c)$ and the derived argon density $(d)$ plotted versus time during the after-puff phase. The bottom trace shows a magnetic pick-up signal $(e)$. The central impurity density was reduced during continuous $n=1$ MHD modes and sawtooth crashes.

density. The beneficial effect of continuous ( $m=1, n=1)$ MHD modes in removing impurities from the plasma core was also reported from ASDEX-Upgrade [24].

The effect of sawteeth and other MHD instabilities upon the impurity density profiles was studied primarily at low to moderate impurity injection rates, where the impurity radiation did not saturate the SXR signal. Radiation fluxes from two SXR cameras were tomographically inverted to deduce the Ar density. The SXR emissivity was assumed to be constant on flux surfaces. The local emissivity, $\varepsilon_{\mathrm{sxr}}$, can be written as a function of impurity density, $n_{\mathrm{I}}$, and the electron density, $n_{\mathrm{e}}$, as:

$$
\varepsilon_{\mathrm{sxr}}=\frac{n_{\mathrm{e}}^{2}}{Z_{D}} L_{D}^{\mathrm{sxr}}+n_{\mathrm{e}} \sum_{I} n_{I}\left(L_{I}^{\mathrm{sxr}}-\frac{Z_{I}}{Z_{D}} L_{D}^{\mathrm{sxr}}\right) .
$$

The first term describes the bremsstrahlung radiation (for zero dilution), while the second term describes the radiation caused by each impurity, $I$, including the dilution of the main ion, $D$. For the plasma core, the mean charge, $Z$, and the total SXR power coefficients, $L^{\mathrm{sxr}}$, of each element mainly depend upon the electron temperature, $T_{\mathrm{e}}$, where deviations from corona ionization equilibrium were determined by the impurity transport code STRAHL [25,37]. The relevant atomic data and the detection efficiency were calculated from tabulated coefficients in the ADAS database [38]. Since the concentration of other impurities is not known very well, $\varepsilon_{\mathrm{sxr}}$ can only be used to deduce Ar densities if SXR radiation of Ar dominates. This is clearly the case, as can be seen in the upper trace in figure 5, where the SXR emission strongly increases due to Ar puff. Thus, the offset radiation was simply estimated by assuming a fixed carbon concentration of $2 \%$ and Ar densities were calculated from the remaining difference from this bremsstrahlung offset.

\section{Impurity peaking and ELM observations}

In the fuelling phase, the combination of Ar and strong deuterium fuelling, a low pedestal pressure was established with a transition from type I to type III ELMs [15]. In the after-puff phase, as the edge cooling was reduced, a transition back into type I ELMs occurred. The type I ELM-frequency decreased as the radiation rate increased [39]. In discharges with high Ar injection (as in figure 1), ELM-free periods (up to $0.5 \mathrm{~s}$ ) were observed. Core impurity accumulation following type I ELMs was evaluated as a contributor to the confinement degradation from bolometry and from SXR data analysis. No correlation between central Ar accumulation and ELM activity was found.

SXR emission in the after-puff phase indicated that core impurity accumulation occurred independent of changes in the accumulation in the outer region (figure 7). The central $\mathrm{Ar}$ density, $n_{\mathrm{Ar}}(0)$, in the after-puff phase increased by $30 \%$ in the discharge with ICRH (from 0 to $1 \mathrm{~s}$ in figure 5), whilst in a similar discharge without added ICRH, $n_{\mathrm{Ar}}(0)$ increased by $120 \%$ (from 0 to $2 \mathrm{~s}$ in figure 7 ). Nevertheless, at a normalized radius of 0.7 , the Ar density was the same in both discharges (figure 7).

The SXR emissivity profiles indicated that the impurity density increased during the ELM-free period following each type I ELM. The increase occurred mostly in the outer region, within $20-30 \mathrm{~cm}$ from the edge (figure 8). During the time interval shown in figure 8 , in the discharge with combined heating, the type I ELM frequency was nearly $30 \%$ higher $\left(f_{\mathrm{ELM}}=36 \mathrm{~Hz}\right.$ in the discharge with ICRH, whilst $f_{\mathrm{ELM}}=$ $26 \mathrm{~Hz}$ in the discharge with $\mathrm{NBI}$ ); however, the region where the SXR increased was similar (figure 8). 

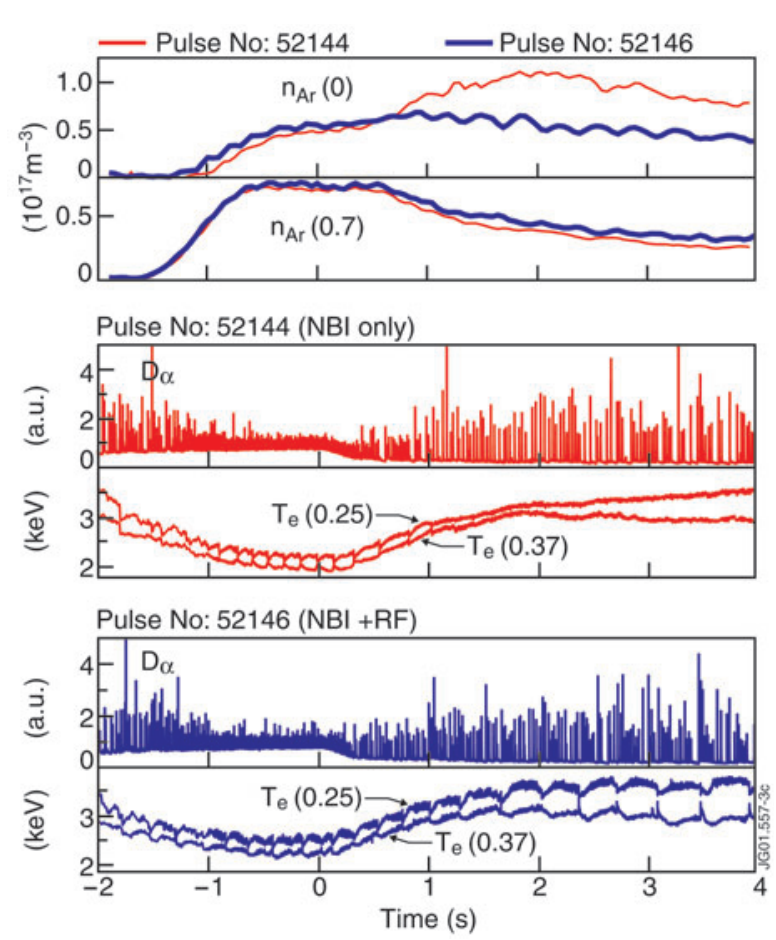

Figure 7. Argon density, $\mathrm{D}_{\alpha}$ emission and electron temperature from ECE heterodyne radiometer measurements at radii outside and inside the sawtooth inversion radius plotted versus time, for discharges $52144\left(P_{\mathrm{NBI}}=12 \mathrm{MW}\right)$ and 52146

$\left(P_{\mathrm{NBI}}=12 \mathrm{MW}+\mathrm{P}_{\mathrm{ICRH}}=3 \mathrm{MW}\right.$, see figures 5 and 6$)$. Time $=0 \mathrm{~s}$ is the start of the after-puff phase. Sawteeth stop at $t=1 \mathrm{~s}$ in the discharge with NBI only, while sawteeth are observed up to the end of the heating phase in the discharge with added ICRH. The average ELM frequencies in the after-puff phase were $f_{\mathrm{ELM}}=30 \mathrm{~Hz}$ in the discharge with NBI only and $f_{\mathrm{ELM}}=45 \mathrm{~Hz}$ in the discharge with added ICRH
Bolometer data analysis indicated that central impurity accumulation in the Ar discharges occurred independent of ELM regime (figure 9). The central Ar accumulation was also independent of ELM amplitude (figure 10), as characterized by the average $\mathrm{D}_{\alpha}$ emission during the ELMs.

\section{Experiments to control $q(0)$ and maintain sawteeth}

To keep $q(0)$ below unity and maintain sawteeth, a small amount of ICRF power was added to the main NBI heating. The ICRH was tuned, on the one hand, to increase the core current density and, on the other hand, to avoid creating the long sawtooth-free periods that are usually observed with ICRH [40]. The RF heating resonance layer was located on-axis to increase the central electron temperature. However, large sawteeth can be created when ICRH heating is applied inside the $q=1$ region. Thus the ICRH power was deliberately kept low (1-3 MW) in order to not create a significant population of ICRF accelerated ions, thus avoiding sawtooth stabilization by fast particles [41]. Hydrogen was used as the minority species, with the antennas operated either in dipole, or with $-\pi / 2$ phasing. In both configurations the central $T_{\mathrm{e}}$ was increased $\left(\Delta T_{\mathrm{e}}(0) \sim 0.5 \mathrm{keV}\right)$, preventing $q(0)$ increasing as fast as in the reference discharges. In these experiments, sawteeth were maintained and the impurity profile did not become as peaked as in discharges without added ICRH (figures 7 and 11).

Experiments with ICRH were performed at different $\mathrm{Ar}$ injection rates. At moderate injection rates $\left(\phi_{\mathrm{Ar}}=\right.$ $2 \times 10^{21} \mathrm{el} \mathrm{s}^{-1}$ in figure 7), although $n_{\mathrm{Ar}}(0)$ increased over $100 \%$ during the after-puff phase, the actual central Ar concentration was negligible $\left(C_{\mathrm{Ar}}(0) \sim 0.1 \%\right.$ for the

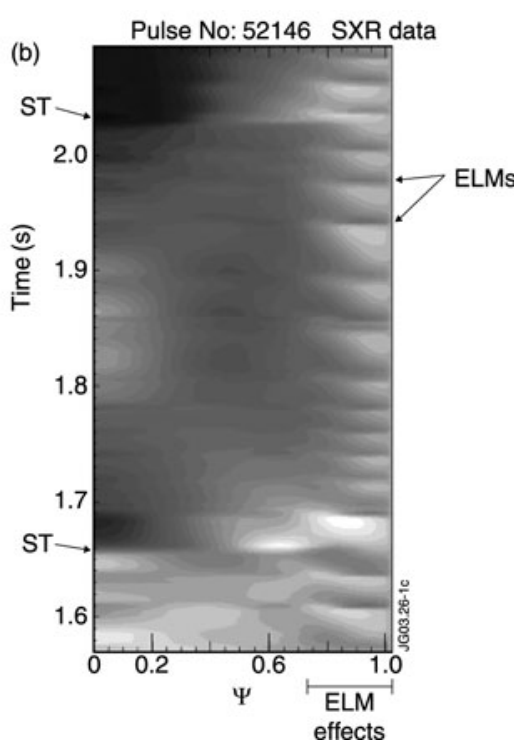

Figure 8. (a) SXR perturbation emissivity profiles (subtracting the average profile) for discharge 52144 (NBI only) after sawtooth suppression. The brightness of this plot varies between $+21.5 \mathrm{~kW} \mathrm{~m}^{-3}$ (white) and $-21.5 \mathrm{~kW} \mathrm{~m}^{-3}$ (black). Following type I ELMs $\left(f_{\mathrm{ELM}}=26 \mathrm{~Hz}\right)$, the perturbation emissivity immediately decreases and an increase is observed between ELMs for $\Psi>0.7$. The central perturbation is related to continuous MHD $(n=3)$ observed after sawtooth suppression (not discussed in this paper). (b) SXR perturbation emissivity profiles (subtracting the average profile) for discharge 52146. In this discharge central impurity accumulation was controlled with ICRH. The brightness of this plot varies between $+9.7 \mathrm{~kW} \mathrm{~m}^{-3}$ (white) and $-9.7 \mathrm{~kW} \mathrm{~m}^{-3}$ (black). The ELM frequency differs $\left(f_{\mathrm{ELM}}=36 \mathrm{~Hz}\right)$ but ELM effects are as in $(a)$. Sawtooth crashes caused the central value to decrease and the $\Psi>0.7$ values to increase. 


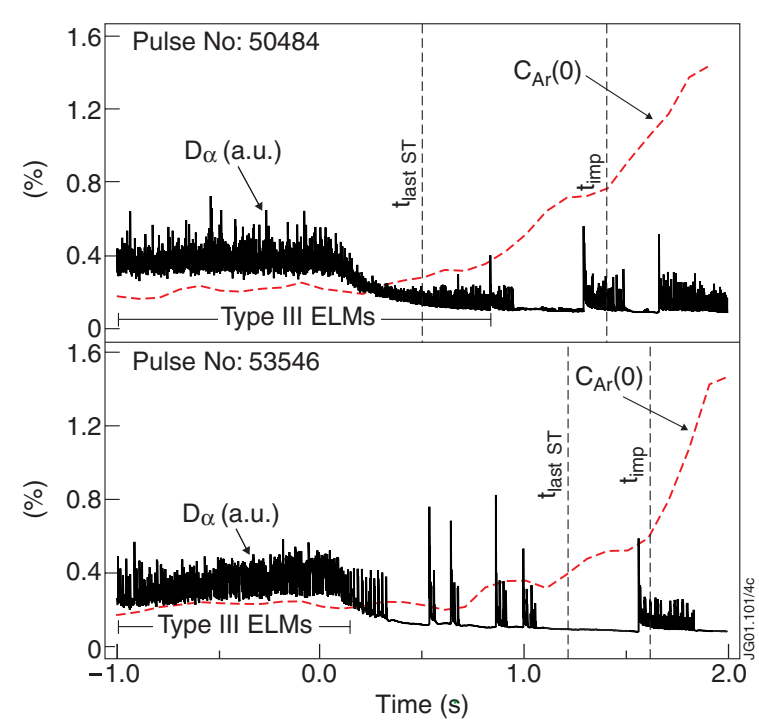

Figure 9. Central impurity concentration $C_{\mathrm{Ar}}(0)$ (calculated from bolometer data analysis) and $\mathrm{D}_{\alpha}$ signal versus time, where $t=0 \mathrm{~s}$ is the start of the after-puff phase for discharges with high Ar injection rates. In the first discharge, the central Ar concentration started to increase in the presence of type III ELMs. In the second discharge, central impurity accumulation started following type I ELMs. Dashed lines indicate: $(a)$ the time the sawtooth instability stopped and $(b)$ the time of sudden impurity increase, defined as in figures 2 and 10 as the time when $\Delta C_{\mathrm{Ar}}(0) / \Delta t \geqslant 1.0 \% \mathrm{~s}^{-1}$.

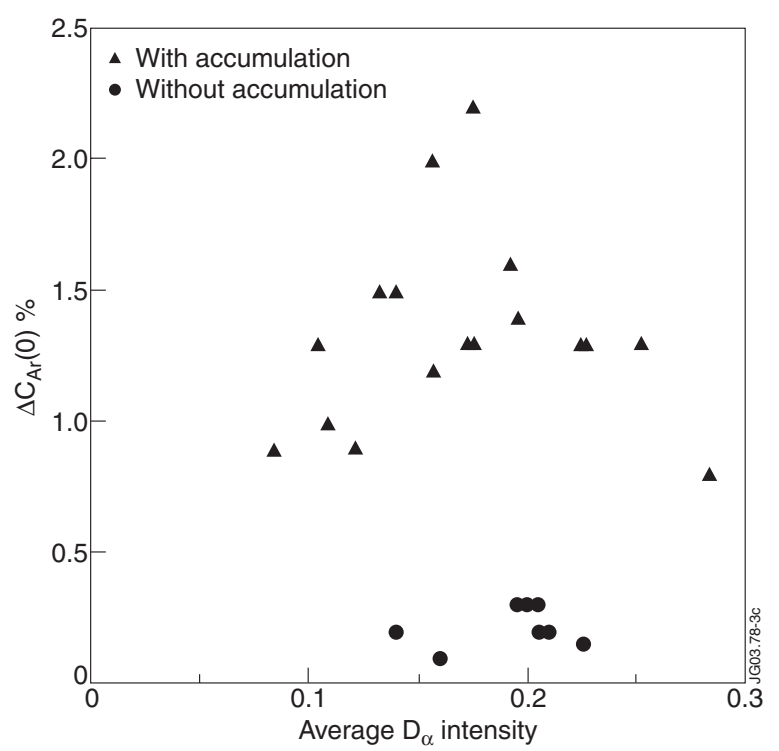

Figure 10. Change in central impurity concentration observed in the after-puff phase $\left(\Delta C_{\mathrm{Ar}}=C_{\mathrm{Ar}}^{\max }-C_{\mathrm{Ar}}\left(t_{\mathrm{imp}}\right)\right)$, plotted versus the average ELM amplitude. The average ELM amplitude is defined as the $\mathrm{D}_{\alpha}$ emission above inter-ELM level, integrated and time averaged from the start of the after-puff phase $(t=0 \mathrm{~s})$ to the increase in central concentration ( $t=t_{\mathrm{imp}}$ in figure 1) for discharges where $C_{\mathrm{Ar}}(0)$ increases at a rate rate $\geqslant 1.0 \% \mathrm{~s}^{-1}$. In the discharges without accumulation $\mathrm{D}_{\alpha}$ was integrated for $1 \mathrm{~s}$.

discharges in figure 7). The benefits of adding ICRH heating were demonstrated at higher injection rates $\left(\phi_{\mathrm{Ar}}=\right.$ $4 \times 10^{21} \mathrm{el} \mathrm{s}^{-1}$ in figures 11 and 12). With ICRH, the central Ar concentration was decreased and maintained at $1 \%$, instead of $2 \%$ without ICRH. Loss of confinement and density were not

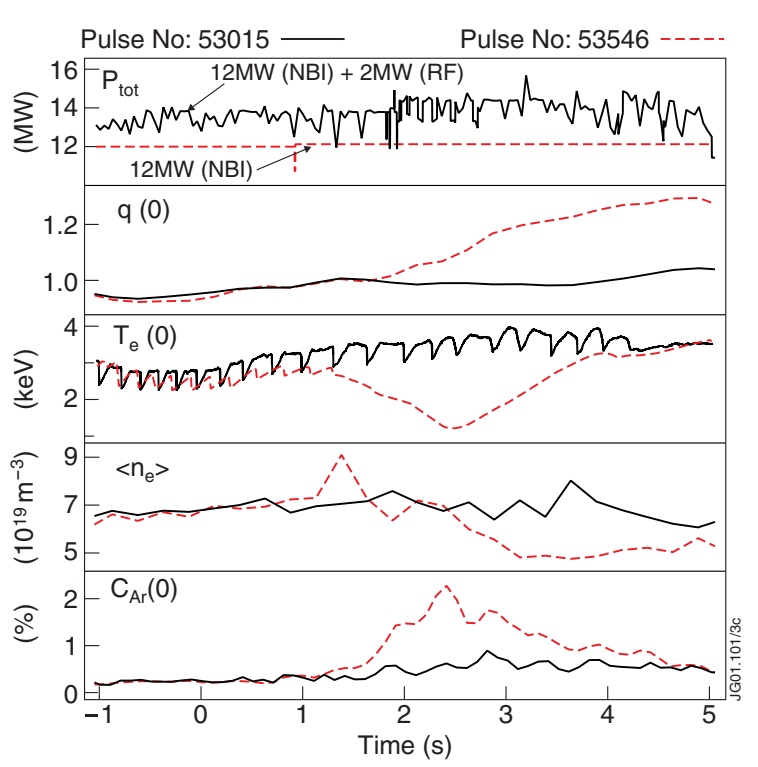

Figure 11. Temporal evolution of $q(0)$, central temperature, $T_{\mathrm{e}}(0)$, average density, $\left\langle n_{\mathrm{e}}\right\rangle$, and Ar concentration, $C_{\mathrm{Ar}}$, for discharges with high Ar injection rates $\left(\phi_{\mathrm{Ar}}=4 \times 10^{21} \mathrm{el} \mathrm{s}^{-1}\right.$ as shown in figure 1), with and without ICRH. Time $=0 \mathrm{~s}$ is the start of the after-puff phase. In the combined heating discharge, $q(0)$ did not increase, sawtooth was maintained and density loss and core impurity accumulation were not observed.

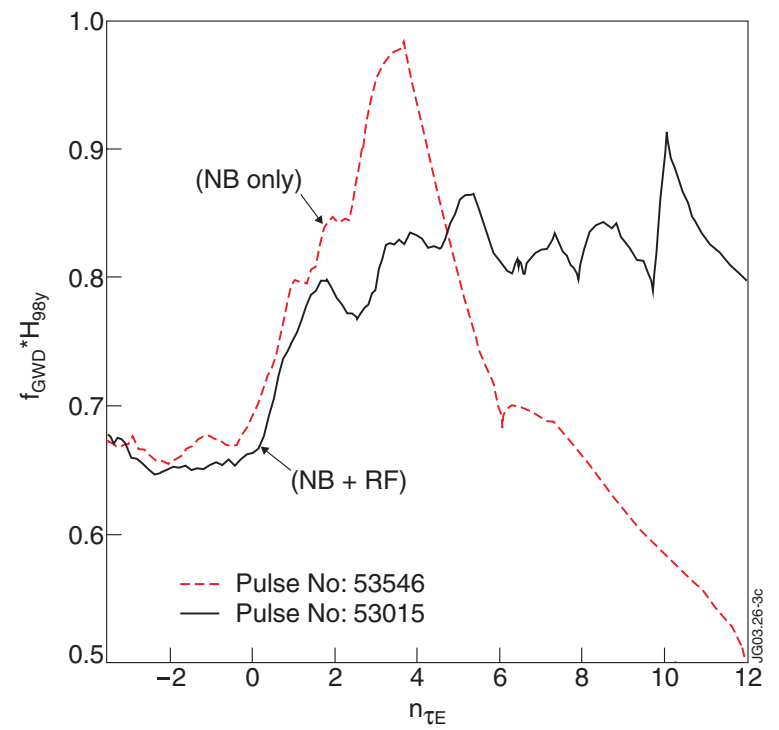

Figure 12. The product $H_{98 y} * f_{\mathrm{GWD}}$ versus the number of energy confinement times, $n \tau_{E}=$ time $/\left\langle\tau_{E}\right\rangle$, for the discharges shown in figure 11. In the discharge with the sawteeth maintained, $H_{98 y} * f_{\mathrm{GWD}} \sim 0.8$ lasted for nine confinement times.

observed (figure 12). Thus in the discharge with added ICRH the high-performance phase was extended (from less than $1 \tau_{E}$ to $9 \tau_{E}$ ) and lasted in a quasi-steady-state until the heating was turned off. In addition, there is no loss of confinement when compared with a non-seeded discharge.

Although adding ICRH has successfully prevented $q(0)$ from rising above unity, it has not restored the peaked current density and the low $q(0)$ values observed in the puffing phase. Magnetic equilibrium calculations and MHD observations 
indicate that with ICRH, $q(0)$ is around unity (with $q$-profiles similar to those shown in figure 4 , top). In addition to the sawtooth instability, a nearly continuous $n=1$ mode was observed (figure 6(e)). SXR data analysis indicated that both the sawtooth crash and the preceding $n=1$ mode prevented impurity profile peaking. On the other hand, flattening of the electron density is also thought to be caused by the presence of the $n=1$ continuous mode. Thus, for effective use of ICRH, the changes in MHD stability, including fast-particle effects, need to be modelled and understood. Although a significant energetic ion component was not expected for densities so close to the density limit, the fast-ion pressure inside the $q=1$ radius increased by $20-30 \%$. Thus fast-ion effects on the internal kink stability may be responsible for the increased sawtooth periods in the discharges with combined heating [41]. (The power deposition of ICRH ions was studied using the analysis codes TRANSP [42, 43] and PION [44, 45].)

\section{Discussion}

Experimental results indicated that sawteeth contributed to impurity control. SXR data analysis showed that central MHD instabilities, sawtooth crashes and, to a lesser extent, continuous $n=1$ modes prevented impurity peaking. However, other mechanisms affecting impurity transport may also have contributed to impurity control. Increasing power in the plasma core may prevent impurity accumulation in several ways. It maintains the central MHD instabilities and their beneficial effect in re-distributing impurities. In addition, a higher temperature would increase Ar ionization, leading to a decreased radiation from the central region. The improved power balance may avoid the impurity accumulative instability as suggested in [19].

The impurity accumulation is often described by neoclassical theory [19]. Usually good agreement is found between the experimental derived transport coefficients and neo-classical theory, in periods between sawtooth crashes [24] and after sawtooth suppression [26]. The flux of the impurity ions, $\Gamma_{Z}$, can be described by diffusive and convective terms: $\Gamma_{Z}=D_{\perp}\left(\mathrm{d} n_{Z} / \mathrm{d} r\right)+V_{\perp} n_{Z}$, where $D_{\perp}, V_{\perp}$ and $n_{Z}$ are, respectively, the impurity perpendicular diffusion coefficient, perpendicular convective velocity and density.

$D_{\perp}$ is the sawtooth-period time-averaged anomalous diffusion coefficient that includes neo-classical theory and sawtooth crash-determined components. In the presence of sawtooth relaxations the diffusive transport is thought to increase due to profile flattening effects during the actual saw tooth crashes. On the other hand, if the convective transport is determined by neo-classical theory only, it is governed by the gradients of the ion density and ion temperature profiles [19].

In the JET experiments considered here, argon was found to be in the Pfirsch-Schlüter collisionality regime. It is shown in [19] that for neo-classical convection in the PfirschSchlüter regime, changes in the background ion density and temperature have opposite effects on the convection velocity. Impurity accumulation may be prevented by an increased $\operatorname{grad}\left(T_{\mathrm{i}}\right)$, referred to as a 'temperature screening'. Impurity accumulation is governed by a competition between the stabilizing effect of impurity diffusion and heat conduction and the destabilizing effect of neo-classical convection and radiation. In the model described in [19], where strong coupling of $T_{\mathrm{e}}$ and $T_{\mathrm{i}}$ is assumed and the plasma heat conductivity, $K_{\perp}$, is taken as $K_{\perp}=3 n_{\mathrm{i}} D_{\perp}$, a critical density criterion for the development of impurity accumulation was given (equation (10) in [19]). This criterion was tested for pulse 46970, where impurity peaking occurred before the end of the puffing phase. The measured density was of the same order as the expected critical density. Increasing heating power in the plasma core would increase the central ion temperature. The temperature screening and the critical density for the accumulation instability would also increase.

In [19] a critical heating power density, $Q_{\text {crit }}$, is introduced, below which a development of the impurity instability is expected: $Q_{\text {heat }} \leqslant Q_{\text {crit }}=C\left(D_{\text {neo }} / D_{\perp}\right) Z_{I} Q_{\text {rad }}$. $Q_{\text {heat }}$ and $Q_{\text {rad }}$ are the central heating power and central radiation power densities. $C$ is a constant of order 1 that depends on profile effects, $Z_{I}$ is the charge of the impurity, in our case of argon not fully ionized, $Z_{I} \approx 16$ (for the $T_{\mathrm{e}}$ observed in the plasma core). (Note: There is a mistake in equation (11) of [19], where $Z_{I}$ is missing in the numerator.)

In order to test this criterion, the power balance in the plasma core was studied (figure 13), for the high Ar rate discharges compared in the previous section (figures 11 and 12). The heating power density was calculated using the analysis code TRANSP $[42,43]$, while the radiative heating density was obtained from bolometry measurements. The critical power density, $Q_{\text {crit }}$, was estimated taking sawtoothaveraged diffusion coefficients $D_{\text {neo }} / D_{\perp} \approx 0.2$ [46]. In the reference discharge (NBI only), $Q_{\text {rad }}$ started to increase slowly at $t \sim 0.75 \mathrm{~s}$. When the sawteeth disappeared, $t>1.3 \mathrm{~s}$, $Q_{\text {crit }}$ exceeded $Q_{\text {heat }}$. Thus the sudden impurity accumulation that followed could be explained on the basis of the instability proposed in [19]. Also as proposed in [19], an increase of the heat power density would prevent impurity accumulation.

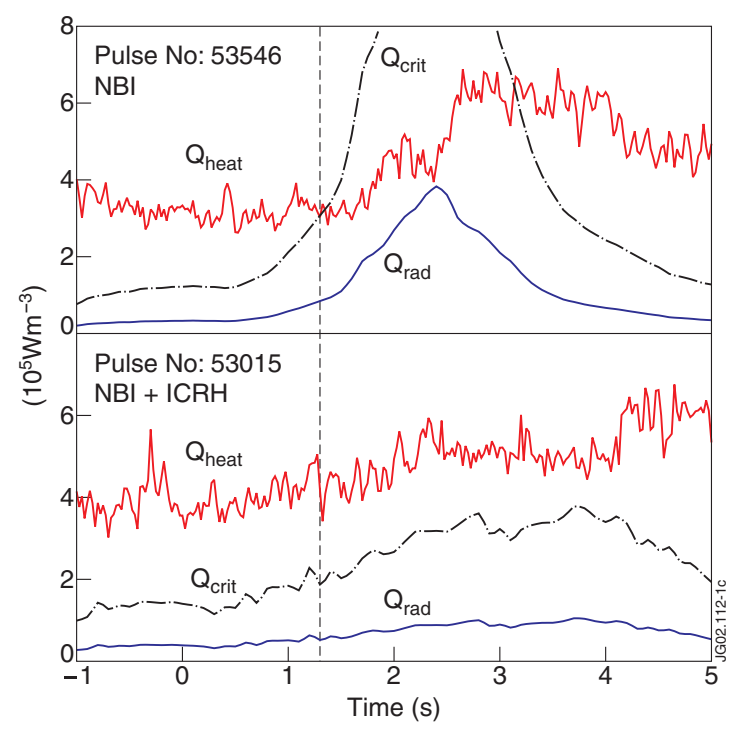

Figure 13. Volume-averaged power densities at $\Psi=0.1$ plotted against time, where $t=0$ is the start of the after-puff phase. $Q_{\text {heat }}$ is the heating power calculated by TRANSP, $Q_{\text {rad }}$ is the radiated power measured by the bolometers. The critical power density, $Q_{\text {crit }}$, was estimated taking diffusion coefficients $D_{\text {neo }} / D_{\perp} \approx 0.2$. Sawteeeth in the discharge with NBI only (top) stopped at $t=1.3 \mathrm{~s}$ (indicated by dotted line, see also figures 1 and 12). 
When the heating power density was increased by ICRH, $Q_{\text {crit }}$ was always below the actual heating power density, $Q_{\text {heat }}$ (figure 13). However, more detailed analysis of the transport coefficients is required, in particular in the ICRH discharges, where doubts remain if the convection velocity is purely neo-classical [46].

Experiments at TEXTOR $[47,48]$ did show a similar beneficial effect of ICRH on the impurity accumulation behaviour. At TEXTOR the transport of tungsten was investigated in neon RI-mode plasmas. Since argon in JET plasmas was in the Pfirsch-Schlüter collisionality regime, the experiments with tungsten (also in the Pfirsch-Schlüter) in TEXTOR RI-mode plasmas are a good comparison. It was found that ICRH leads to a broadening of electron density profiles and consequently to an outward directed neo-classical convective velocity, which suppresses accumulation effects. This is very similar to the convective velocity of argon in JET, which is also outward directed in discharges with ICRH [46].

One advantage of increased heating was to avoid the radiative collapses that are sometimes observed. These limit the amount of deuterium and argon that can be used in the afterpuff phase and may lead to disruptions. In figure 13, the core radiation is approximately equal to the local heating power in the after-puff phase in the discharge with NBI heating. This local radiative collapse was not observed with added ICRH.

Power scans planned for future JET experiments will assess the advantages of increasing power with ICRH in comparison with increased NBI heating. A discharge with an NBI power of $14 \mathrm{MW}$ was produced for comparison with the combined heating discharge discussed above (figures 11-13). In this higher power, NBI-only discharge, no impurity accumulation was observed, in spite of sawtooth suppression. However, the sawtooth was substituted by a continuous central $n=1$ mode that remained until the end of the heating phase. SXR analysis indicated that continuous central modes helped to prevent impurity accumulation. The effect of MHD modes on impurity transport coefficients needs to be better understood. In addition, this particular discharge had a central electron density $30 \%$ lower than in the discharges discussed above, making the impurity transport not easily comparable.

Larger ICRH powers, leading to $T_{\mathrm{e}}(0)>5 \mathrm{keV}$, would imply a higher ionization degree for the impurities in the plasma core, with a large percentage of fully stripped $\mathrm{Ar}$ ions. In this situation, the same Ar concentration would correspond to a decreased radiation from the centre (by a factor of about 2 with $T_{\mathrm{e}}(0)=6 \mathrm{keV}$ at the same electron density) [46]. Furthermore, at higher temperatures, argon is in the banana-plateau regime. In this case the proposed accumulation instability [19] cannot develop, since the balance between convective and diffusive transport contributions to the neoclassical transport will change. This is mainly due to the fact that $D_{\text {neo }}$ decreases by going to higher temperatures and the effect of neo-classical impurity transport is weaker.

\section{Conclusions}

Loss of confinement in low-triangularity discharges with high Ar injection rates was correlated with impurity accumulation in the plasma core. This was usually observed in the after-puff phase. Both edge and central MHD activities were assessed as contributors to performance degradation. No correlation was found between central impurity peaking and ELM activity. There was, however, a correlation between impurity density peaking and absence of sawteeth MHD activity. SXR data analysis indicated that the impurity density profile was flattened during sawteeth crashes. The impurity inflow into the central region was also reduced by continuous $n=1$ MHD modes observed when $q(0)$ is close to unity $(q(0) \sim 1)$. Sawteeth were suppressed when $q(0)$ rose above unity. After sawteeth suppression, reversed shear $q$-profiles were obtained.

Maintaining sawteeth by using ICRH resulted in quasisteady-state, high-performance plasmas with high Ar densities. Values of $H_{98 y} * f_{\mathrm{GWD}} \sim 0.8$, previously only lasting less than $1 \tau_{E}$ at high $\mathrm{Ar}$ injection rates, were now maintained for the duration of the heating phase $\left(\Delta t \sim 9 \tau_{E}\right)$. These experiments lead to the conclusion that the sawtooth, together with other central MHD instabilities, played an important role in preventing central impurity accumulation. The increased central heating maintains sawtooth MHD activity, keeps $q(0)$ stationary and possibly opposes impurity accumulation by changing the core power balance and modifying the impurity transport as predicted by neo-classical theory. A combination of MHD and temperature screening effects may have prevented impurity accumulation in discharges with added ICRH.

Future experiments and modelling should assess the role of increased central heating on impurity transport. In particular, it is necessary to find out how impurity transport coefficients are altered by sawtooth and other central MHD modes. Estimates of impurity transport coefficients [46] indicated that with ICRH the impurity convective velocity is directed outward, which is consistent with neo-classical transport in the Pfirsch-Schlüter regime with a peaked temperature profile.

In this paper, ICRH has been used to control the $q$ profile, sawtooth instability and impurity accumulation in low triangularity plasmas. Recently, these techniques have been extended to high-triangularity $(\delta>0.4-0.5)$, high-density, ELMy H-mode plasmas both with and without impurity injection. ICRH is now used routinely in impurity injection experiments in ITER-like configurations to increase discharge stationarity. Also, ICRH-controlled impurity accumulation was associated with sawtooth loss in recent JET experiments exploring a type I-type II ELM regime in ITER-like and quasidouble-null configurations $[49,50]$.

\section{Acknowledgments}

The authors are grateful to Dr M. Tokar and Dr O. Sauter for useful discussions. This work was performed under the European Fusion Development Agreement. It received financial support from Fundação para a Ciencia e Tecnologia (FCT), Portugal. The content of the publication is the sole responsibility of the authors and it does not necessarily represent the views of the Commission of the European Union or FCT or their services.

\section{References}

[1] Weynants R.R. et al 1999 Nucl. Fusion 391637

[2] Vandenplas P.E. et al 1998 J. Plasma Phys. 59587 
[3] Wolf G.H. et al 1997 Proc. 16th Int. Conf. on Fusion Energy 1996 (Montreal, 1996) vol 1 (Vienna: IAEA) p 177

[4] Jackson G.L. et al 1999 J. Nucl. Mater. 266-269 380

[5] McKee G.R. et al 2000 Phys. Rev. Lett. 841922

[6] Murakami M. et al 2001 Nucl. Fusion 41317

[7] Jackson G.L. 2002 Nucl. Fusion 4228

[8] Ongena J. et al 2001 Plasma Phys. Control. Fusion 43 12A

[9] Dumortier P. et al 2002 Plasma Phys. Control. Fusion 441845

[10] Maddison J. et al 2003 Nucl. Fusion 4349

[11] Kubo et al 2000 Proc. 18th Int. Conf. on Fusion Energy 2000 (Sorrento, 2000) (Vienna: IAEA) CD-ROM file and http://www.iaea.org/programmes/ripc/physics/fec2000/ $\mathrm{html} /$ node $1 . \mathrm{htm}$

[12] Hill K.W. et al 2002 Proc. 19th Int. Conf. on Fusion Energy 2002 (Lyon, 2002) (Vienna: IAEA) CD-ROM file and http://www.iaea.org/programmes/ripc/physics/fec2002/ $\mathrm{html} / \mathrm{fec} 2002 . \mathrm{htm}$

[13] ITER Physics Basis 1999 Nucl. Fusion 392175

[14] Jackson G.L. et al 2002 Plasma Phys. Control. Fusion 441893

[15] Strachan J. et al 2000 Plasma Phys. Control. Fusion 42 A81

[16] Keilhacker M. and the JET Team 1999 Nucl. Fusion 39209

[17] Koslowski R. et al 1997 Plasma Phys. Control. Fusion 39 B325

[18] Koslowski H.R. et al 2001 Proc. 28th EPS Conf. on Controlled Fusion and Plasma Physics (Funchal, 18-22 June 2001) vol 25A (ECA) 965

[19] Tokar M.Z. et al 1997 Nucl. Fusion 371691

[20] Zohm H. 1996 Plasma Phys. Control. Fusion 38105

[21] Post et al 1977 Atomic Data Nucl. Data Tables 20397

[22] de Vries P. et al 2001 Proc. 28th EPS Conf. on Controlled Fusion and Plasma Physics (Funchal, 18-22 June 2001) vol 25A (ECA) 1777

[23] Gehre O. et al 1987 Proc. 14th European Conf. on Controlled Fusion and Plasma Physics (Madrid) (part I) p 156

[24] Dux R. et al 1999 Nucl. Fusion 391509

[25] Guenter S. et al 1999 Nucl. Fusion 391535

[26] Rapp J. et al 1997 Plasma Phys. Control. Fusion 391615

[27] Lao L.L. et al 1990 Nucl. Fusion 301035
[28] Braithwaite G. et al 1989 Rev. Sci. Inst. 602825

[29] Gianella R. et al 1994 Nucl. Fusion 341185

[30] Pasini D. et al 1990 Nucl. Fusion 302049

[31] Seguin F., Petrasso R. and Marmar E.S. 1983 Phys. Rev. Lett. 51455

[32] Petrasso R. et al 1982 Phys. Rev. Lett. 491826

[33] Hinnov E. et al 1980 Bull. Am. Phys. Soc. 25902

[34] Ida K. et al 1987 Phys. Rev. Lett. 58116

[35] Ida K. et al 1986 Plasma Phys. Control. Fusion 28879

[36] Pasini D. et al 1992 Plasma Phys. Control. Fusion 34677

[37] Dux R. and Peeters A.G. 2000 Nucl. Fusion 40 1721-9

[38] Henke B.L., Gullikson E.M. and Davis J.C. 1993 Atomic Data Nucl. Data Tables 54

[39] Jachmich S. et al 2002 Plasma Phys. Control. Fusion 441879

[40] Campbell D.J. et al 1988 Phys. Rev. Lett. 602148

[41] Porcelli F., Boucher D. and Rosenbluth M.N. 1996 Plasma Phys. Control. Fusion 382163

[42] Goldston R.J. et al 1981 J. Comput. Phys. 4361

[43] Budny R.V. 1994 Nucl. Fusion 341247

[44] Erikson L.-G., Hellsten T. and Willen U. 1993 Nucl. Fusion 33 1037

[45] Erikson L.-G. and Hellsten T. 1995 Phys. Scr. 5570

[46] Puiatti M.E. et al 2002 Plasma Phys. Control. Fusion 441863

[47] Rapp J. et al 1998 2nd Europhysics Topical Conf. on RF Heating and Current Drive of Fusion Devices (Brussels, Belgium, 20-23 January 1998) (Europhysics Conference Abstracts) vol 22A, p 89

[48] Van Oost G. 1995 22nd EPS (part III) p 345

[49] Saibene G. et al 2003 Pedestal and ELM characterization of highly shaped single null and quasi-double dull plasmas in JET, at high-confinement and density 30th EPS Conf. on Controlled Fusion and Plasma Physics (Petersburg, 7-11 July 2003)

[50] Sartori R. et al 2003 Interaction between core and edge in high density JET Elmy H-modes with good confinement and extension of this regime towards high plasma current 30th EPS Conf. on Controlled Fusion and Plasma Physics (Petersburg, 7-11 July 2003) 